\title{
DESARROLLO PERSONAL, PROFESIONAL E INSTITUCIONAL Y FORMACIÓN DEL PROFESORADO. ALGUNAS TENDENCIAS PARA EL SIGLO XXI
}

\author{
Francisco Imbernón* \\ Universidad de Barcelona
}

\section{RESUMEN}

El artículo analiza la formación y el desarrollo del profesorado. Considerando la formación una parte importante de ese desarrollo, pero no la única. El desarrollo docente se ocasiona por diversos componentes que van impactando en el profesorado a lo largo de su vida. Pero no únicamente un desarrollo profesional sino la importancia de desarrollar el componente personal e institucional. Ese desarrollo se realiza en tres etapas, la formación inicial, la inducción a la práctica y la formación y el desarrollo a lo largo de la vida. Y el artículo propone diversas alternativas actuales que permiten un mejor desarrollo personal, profesional e institucional. La mejora del desarrollo del profesorado es un conjunto de factores que posibilitan, o que impedirán o favorecerán, que el profesorado progrese en el ejercicio de su trabajo, en su bienestar docente y mejore el aprendizaje del alumnado.

Palabras Clave: desarrollo profesional docente, profesorado, formación inicial, inducción profesional, formación permanente.

\section{PERSONAL, PROFESSIONAL AND INSTITUTIONAL DEVELOPMENT AND TEACHER TRAINING. SOME TRENDS FOR THE 21ST CENTURY}

\section{Abstract}

The article analyses the training and development of teachers. Considering training an important part of that development, but not the only one. Teaching development is caused by various components that influence teachers throughout their lives. However, not only professional development but also the importance of developing the personal and institutional component. This development is carried out in three stages, the initial formation, the induction to the practice and the formation and the development throughout the life. In addition, the article proposes several current alternatives that allow a better personal, professional and institutional development. The improvement of the development of the teaching staff is a set of factors that make it possible, or that will prevent or favour, that the teaching staff progress in the exercise of their work and improve the learning of the students.

KEYWORDs: teacher professional development, teaching staff, initial training, professional induction, permanent teacher training. 


\section{DESARROLLO Y FORMACIÓN}

Nadie duda que a lo largo de los últimos ańos la formación y el desarrollo profesional del profesorado ha sido una gran preocupación en el campo social y educativo. Solo falta buscar en bases de datos o en las redes para comprobar la cantidad de publicaciones, informes nacionales e internacionales, congresos y jornadas sobre la temática para comprobar que es cierta esa preocupación (Eurydice, 2013; Talis, 2014, 2018; OCDE, 2005). Otro aspecto es el valor real y la aplicación de todo ello, sobre todo en las políticas y prácticas de formación docente, que, desde 2012, fueron recortadas drásticamente en todo el país y que aún perduran instaladas en la administración pública. Y que, hoy día, aún duran sus secuelas y consecuencias.

Ya no estamos es la famosa década de los años 80, década llamada de la formación permanente sobre todo por la aparición de muchos textos anglosajones, cuestionamiento y una cierta formación y propuestas de nuevas organizaciones y alternativas. En el momento actual se tienen otras preocupaciones (metodologías, currículum, organización, evaluación, innovación...), pero, a pesar de ello, el tratamiento de la formación y el desarrollo docente continúa estando en la preocupación de algunas instituciones y muchos docentes.

Como ya he desarrollado en otro artículo (Imbernón, 2002), a menudo empleamos el concepto de formación y el de desarrollo profesional como sinónimos con una equiparación total en sus términos y contenido, haciendo del desarrollo profesional del profesorado una mirada muy restrictiva ya que significaría que la formación es la única vía de desarrollo profesional del profesorado (y por tanto la culpable si no funciona el proceso educativo). Y no es cierto que el desarrollo profesional del profesorado se deba únicamente al desarrollo provocado por la formación. Ya que en el desarrollo docente entran cuestiones de desarrollo personal (vida, situaciones personales, relaciones sociales, afectivas...), estrictamente profesional (formación, salario, carrera docente, alumnado, clima laboral...) e institucional (normativas, estructuras organizativas, clima laboral, jerarquías...) que permiten o impiden el desarrollo de una, adecuada o no, carrera docente a lo largo de la vida.

Por tanto, el desarrollo docente del profesorado es un conjunto de factores que posibilitan, o que impedirán o favorecerán, que el profesorado progrese en el ejercicio de su trabajo. Es cierto que una mejor formación facilitará sin duda ese desarrollo, pero la mejora de los otros factores (salario, estructuras, niveles de decisión, niveles de participación, carrera, clima de trabajo, legislación laboral...) también lo harán y de forma muy decisiva como se comprueba en muchos países donde la situación laboral del profesorado perjudica al componente personal e institucional de su trabajo en la escuela. Se puede tener mucha formación y ejecutar poco cam-

* Universidad de Barcelona, paseo de la Vall Hebrón, 171. 08035 Barcelona. E-mail: fimbernon@ub.edu. 
bio en las prácticas educativas o ir decayendo profesionalmente, por las otras cuestiones laborales, en una proletarización o en un desencanto realizando una educación más por cuestiones administrativas que educativas.

Por tanto, la formación es muy importante, pero el desarrollo global del profesorado es un proceso de mejorar la práctica laboral, las creencias y los conocimientos profesionales a partir del desarrollo como persona, como profesional o trabajador y como integrante en una estructura y organización educativa.

\section{LA PARADOJA ENTRE LO QUE SE DICE Y SE ESCRIBE Y LA REALIDAD PRÁCTICA}

Quizás una de las preocupaciones más importantes es que se escribe y se habla, desde hace muchos años, en conferencias, seminarios o reuniones, del profesorado reflexivo, crítico, investigador u otras adjetivaciones y la práctica de la formación, en todas sus etapas, puede continuar con un desarrollo implícito de racionalidad técnica, lo que significa que puede continuar en un modelo de entrenamiento o de «ignorancia del profesorado» donde se les ha de iluminar cognitivamente del conocimiento dado por otros, o no contar con ellos y ellas y sus situaciones problemáticas. Hablar, escribir, formar al profesorado sin el profesorado ni sobre los saberes necesarios para la práctica educativa en los diversos contextos.

Y en muchos procesos de formación, soportar la incomodidad de prácticas formativas aburridas basadas en procesos de expertos (donde se pretende "culturizar» profesionalmente) que intentan solucionar los problemas genéricos del profesorado. Y que aburren con un exceso de presentaciones expuestas y leídas. Aunque se hable o se ponga como título «ser los protagonistas del cambio». Y eso se puede comprobar en la cantidad de cursos estándar que aún se programan o con tantos expertos (algunos gurús de las redes con pretensión más de vender que de ayudar o transformar) que continúan explicando (no compartiendo) lo que ellos saben o leen (o captan del google) y no lo que parte de las necesidades sentidas de los docentes (las situaciones problemáticas). Aunque se ha de reconocer, desde hace tiempo, que existe una crítica y un cuestionamiento a ese proceso alienador y de domesticación docente y la realización de prácticas de formación alternativas.

Quisiera desgranar lo que sabemos que funciona mejor, dentro de la incertidumbre que caracteriza a las prácticas educativas, y analizar qué provoca ese modelo alienador de entrenamiento formativo y proponer algunas novedades que han ido apareciendo en los últimos decenios del siglo xx y principios del XxI, que pueden posibilitar una mejor profesionalización. También desearía evitar las frases hechas, los textos ya dichos y repetidos hasta la saciedad y los temas más obvios con citas rimbombantes, aunque sean repetidos por ponentes en magníficos power-points o vídeos en Youtube.

Si analizamos el desarrollo profesional del docente, encontramos diferencias, pero complementarias en tres etapas formativas, el famoso llamado contínuum: la entrada en las universidades, donde se inicia la socialización profesional; la etapa de inserción laboral, o sea, la inducción profesional; y la etapa del desarrollo profesio- 
nal continuo, o sea, la formación permanente en el contexto social y laboral. Miremos de analizar las actuales tendencias y preocupaciones de cada etapa.

\section{3. ¿QUÉ HA IDO APARECIENDO EN LOS ÚLTIMOS AÑOS QUE POSIBILITA LA MEJORA DEL DESARROLLO DOCENTE MEDIANTE LA FORMACIÓN INICIAL?}

\section{A) El PRINCIPIO DE LA SOCIALIZACIÓN Y DESARROLLO DOCENTE: LA FORMACIÓN INICIAL}

Respecto a la formación inicial del profesorado sí que ha habido en los últimos años una gran efervescencia en su análisis, cuando en las etapas anteriores no era tan importante o, al menos, no había tanto debate sobre ella. Y aparecen nuevas preocupaciones y debates.

El tema de la formación inicial del profesorado no universitario siempre ha sido una cuestión discutida y bastante analizada. Hay muchos informes, seminarios, grupos de trabajo autonómicos y variedad de propuestas y estudios anteriores (Eurydice, 2002-2006, 2012, 2013; MEC, 2012; Talis, 2014). Pero parece que ahora mucho más, ya sea por un libro blanco aparecido de improviso y encargado por el Gobierno del Partido Popular, que está lleno de opiniones más que de evidencias y que no llegó a buen término (Marina, 2015), o por la preocupación que muestran los informes europeos.

En primer lugar, aparece una preocupación por el acceso a la profesión del profesorado (Vezub, 2005). O sea, si todos los que solicitan un acceso a esta carrera docente tienen las competencias necesarias para ser docente. Aunque es un tema dudoso y discutido sobre si se puede limitar el acceso a la profesión basándose en algunas pruebas, lo que es cierto es que aparece últimamente en todos los debates sobre la formación inicial y algunas autonomías ya realizan pruebas previas de selección posteriores a las pruebas de selección universitarias.

Se argumenta, aunque no esté comprobado por evidencias, que la selección de entrada a los estudios de profesorado aumentará la calidad de la educación. Se quiere incorporar a candidatos y candidatas que posean competencias para esta profesión mediante pruebas específicas sobre todo en lengua y matemática. Es un tema que necesita una mayor reflexión ya que si, por el número de alumnos y alumnas que demandan la carrera de profesorado, no se permite hacer una entrevista personalizada como en otros países y comprobar otras competencias importantes en el desarrollo del profesorado, podemos perder futuros profesores y profesoras que han tenido algún error en pruebas de papel y lápiz. Muchas veces las limitaciones hacen que lo que parece ser una adecuada solución o propuesta se convierta en algo que no provoca lo que se pretendía.

Cierto que todo lo que tiene que ver con la formación inicial del magisterio siempre ha sido un tema controvertido y paradójico desde sus inicios. Controvertido por la diferencia de modelos de formación a nivel internacional y la paradoja radica en que, de manera constante, se ha calificado como una formación escasa, que no 
significa mala sino, en algunos aspectos, inadecuada y, por otro lado, se insta siempre a su mejora, pero se hace muy poco en la realidad de las políticas gubernamentales o universitarias. No importa la persistente recomendación de los informes internacionales que aseguran que su formación es muy importante para el desarrollo del futuro del profesorado y, como consecuencia, para la calidad de la enseñanza y, por lo tanto, para la mejora del aprendizaje del alumnado (Talis, 2018).

Otra preocupación es la adecuación de los estudios a la realidad social, científica y pedagógica actual. Es cierto que formar a futuros profesores y profesoras ha de incluir una sólida formación pedagógica y práctica que posibilite el trabajo educativo con la realidad social que hoy se tiene, diferente a la de hace ańos. Pero a veces no queda más que como reflexión o con propuestas que no se tienen en cuenta ya que eso llevaría a una profunda modificación curricular y organizativa.

Pero la estructura curricular que aún perdura contiene muchos componentes de racionalidad técnica donde el futuro profesor o profesora recibe un conocimiento fraccionado del conocimiento y una receptología de lo que pasará cuando se incorpore a la práctica profesional (Guerra y Lobato, 2015). Y, desde hace años, sabemos que eso no forma a futuros profesores preparados para las circunstancias actuales. Y aparece, cuando se incorpora a la práctica profesional, el famoso choque de la realidad o los sueños rotos (Friedman, 2004), muy investigados hace años pero que no repercuten en el cambio curricular que debería hacerse.

Sería necesario no dar tanta importancia a los contenidos disciplinares (con la conocida asignación curricular de lengua y su didáctica en lugar de didáctica de la lengua u otras áreas de conocimiento de didácticas específicas) y dar mucha mayor importancia a tratar de analizar qué significa la innovación educativa en los centros, cómo se realiza el trabajo en equipo y la colaboración y qué se puede hacer en la educación en las nuevas exigencias culturales, sociales y psicopedagógicas. Y, por supuesto, una trama curricular que permitiera tratar muchas más cosas: la igualdad entre los sexos, la atención a la diversidad, los contextos multiculturales, las ciudadanías democrática, solidaria, medioambiental y un largo etc.

Si la gramática del sistema curricular tiene grandes fallos, la preparación del profesorado lo padece. Quizás se tendría que establecer como una espiral que tuviera como eje central la práctica educativa dando a los temas disciplinarios la incidencia en la reflexión en esa práctica y una mayor flexibilidad curricular adecuada a los diversos contextos. Sería necesario que los futuros docentes puedan recibir una visión holística y crítica de las materias, ya sean de contenido científico o psicopedagógico, a partir del conocimiento y la reflexión sobre la teoría y la práctica docente (González Sanmamed y Fuentes, 2011).

Y no olvidemos la importancia que tienen aquellos y aquellas que forman a los futuros profesores. Algunos con buena intención y creando alternativas docentes, otros con metodologías obsoletas y muchos sin conocer la cultura del magisterio y las circunstancias actuales educativas.

Y quisiera tratar un tema controvertido que aparece a veces como el concepto de "la mística del magisterio", a veces como una mística de servicio herencia de la antigua formación docente. Ello, según algunos autores (Bravo, 2007), impregnaba las escuelas normales que aún existen en algunos (pocos) países latinoamerica- 
nos. Nadie duda que estudiar para ser profesor o profesora ha de tener ciertas consideraciones de ir más allá de los conocimientos disciplinares de forma repetitiva y rutinaria. Ser culto, trabajar temas literarios, música, artes plásticas y escénicas, etc. Son fundamentales. Un magisterio culto es muy importante para el sistema educativo. Un magisterio inculto seguirá las directrices de los demás, será más vulnerable al entorno político y social y utilizará el libro de texto único en su enseñanza o copiará de otros. La mística se traduce hoy día por la cultura docente, o sea, una forma de entender la formación del magisterio, más allá de aprender a enseñar y más aprender a compartir, aprender a aprender, a desaprender y a ver el mundo de una determinada forma para ayudar a cambiarlo y cuestionar sus grandes problemas sociales: democracia, solidaridad, violencia de género, medio ambiente, etc. (Darling-Hammond, 2001).

La formación inicial debería proporcionar una formación humana integral que fomentara las destrezas de pensamiento, el desarrollo de los valores humanos, la ética, la cultura cívica, la moral, la diversidad, la justicia y la crítica. Una sólida preparación en la estructura sustantiva y sintáctica de las disciplinas que se van a enseñar en la práctica educativa y que proporcione una apertura a la investigación en estos campos del saber cuando se desarrolle en la práctica educativa y en contextos específicos.

También conocimientos pedagógicos basados en el rigor y la evidencia de los actuales desarrollos de los campos de conocimiento de las materias educativas. Aprender, analizar experiencias, preguntar y proponer innovaciones. Asumir una actitud hacia el cambio y la innovación.

Aprender con una metodología que desarrolle la colaboración (hoy en día las redes son imprescindibles), la crítica, el trabajo con la comunidad, aprender entre iguales, la comunicación de los problemas, el uso de las tecnologías, los proyectos de trabajo, etc. Una metodología que posibilite desarrollar a un práctico reflexivo más que a un profesor normativo, técnico y sumiso. Debería darse más importancia a la reflexión y la formación sobre los aspectos éticos, relacionales, colegiales, actitudinales, emocionales del profesorado, que van más allá de los aspectos puramente técnicos y «objetivos» de los conocimientos académicos.

Por lo tanto, todo esto nos tendría que traer a fomentar la investigación, la reflexión y la crítica, desarrollar los saberes mínimos requeridos para el inicio de la enseñanza, para evitar la angustia de la entrada a la profesión, este choque con la realidad, aprender cómo desarrollarse en la atención a la diversidad y todo lo que esto implica (justicia educativa), acercar la relación teoría-práctica y situar la práctica docente como eje nuclear de la formación, entre otras cosas (Tardif, 2004), como hemos comentado anteriormente.

B) LA INDUCCIÓN PROFESIONAL EN LA PRÁCTICA LABORAL

Y aparece la segunda etapa del desarrollo: el tema de la inserción en la práctica laboral mediante la inducción profesional.

Es cierto que hace tiempo se trataba el tema (Allen, 2009, Marcelo, 2009). No hay acuerdo en el tiempo y, desde hace tiempo, se realiza de forma diferente en 
diversos países. Se coincide en que tendría que centrarse en una formación práctica con tutores seleccionados y dar valor a la experiencia docente en las instituciones educativas y convertir esa experiencia en una oportunidad de aprender porque son muy importantes las primeras incursiones en la práctica con la problemática que comporta, ya estudiadas desde hace mucho tiempo. Quedan pendientes muchos temas respecto a la inducción profesional: si la fase de inmersión del profesorado novel en la práctica será evaluada, su nivel salarial, el tiempo de docencia, el papel de las universidades, la formación de los tutores, etc. Nadie duda de la importancia de la inducción profesional en el contexto laboral, pero la aplicación práctica comporta ciertas condiciones que no son fáciles de llevar a cabo en todos los países ya que si se realiza una inducción profesional sería necesario la modificación de la entrada en la función pública ya que no es admisible que se vuelva a preguntar en unas pruebas estandarizadas sobre los conocimientos ya evaluados durante la formación inicial docente, y que los ejercicios y supuestos prácticos se obtienen a través de respuestas teóricas que no tienen nada que ver con la práctica real.

\section{EL DESARROLLO DEL PROFESORADO A LO LARGO DE LA VIDA PERSONAL, PROFESIONAL E INSTITUCIONAL}

Respecto a la formación permanente o el desarrollo profesional continuo, hace tiempo ya comentaba algunos aspectos a tener en cuenta en ese proceso de formación en los tiempos actuales (Imbernón, 2001), adaptado y actualizado en este artículo.

La formación permanente del profesorado es una necesidad creciente y es reconocida en documentos y normativas como un derecho y un deber que se extiende a todas las funciones que pueden ejercer los docentes. Y tiene que formar parte intrínseca de la profesión introduciendo esta necesidad perentoria desde los inicios de los estudios de profesorado.

Y desde hace tiempo se viene investigando y analizando qué tipo de formación permanente permitirá un mejor desarrollo docente y qué tendría que modificarse para realizar una diferente formación permanente. Parece que últimamente continúa debatiéndose, pero se ha ido diluyendo. Analicemos por qué. Una formación permanente del profesorado que empezó con entusiasmo en 1984 (la década de los 80 fue la década en muchos países de la formación permanente del profesorado) con la creación de los centros de profesores y se paralizaban las actividades centralizadas de los institutos de ciencias de la educación de las universidades que lo habían ido haciendo desde su creación en la década de los años 1970 del anterior siglo.

Fue una época donde se implicaron movimientos, sindicatos y profesorado para mejorar la formación. Se formaron asesores (Alen, 2009), se hicieron experiencias muy interesantes y se teorizó mucho, tanto por el profesorado universitario como por el no universitario.

Ese entusiasmo tenía importantes motivos ya que se descentralizaba la formación en los territorios, se creaban figuras como la de asesoramiento y se iba intro- 
duciendo la formación en centros cuyas características eran: una formación en el centro o en el territorio, partir de las necesidades sentidas docentes a partir de las situaciones problemáticas de los contextos, con una participación del profesorado en todo el proceso formativo: planificación, desarrollo y evaluación de la formación. Fue un importante revulsivo para iniciar un cambio en la formación permanente.

Una formación basada en las situaciones problemáticas del profesorado que se daban en la institución educativa, con intención de superar los procesos formativos estándar que trataban la formación como un problema genérico que resolvían expertos y diseminaban para todo el profesorado en cursos o seminarios. La lógica de esa forma de tratar el proceso de la formación ha sido históricamente el positivismo, una racionalidad técnica que buscaba con ahínco acciones generalizadoras para llevarlas a los diversos contextos. Una verdadera falacia. La formación intentaba dar respuesta, sin éxito, a ese problema común. La formación más basada en situaciones problemáticas intenta dar la palabra a los protagonistas de la acción, empoderarlos de su propia formación y mejorar el desarrollo de la innovación institucional en los centros. La formación necesita pasar del desarrollo de la experiencia de innovación aislada a una innovación institucional colectiva que se consolida en las instituciones educativas.

Pero ya sabemos que las políticas públicas condicionan la formación. Una determinada ideología más progresista origina procesos más cercanos a los docentes y una formación más horizontal, con cierta confianza en ellos, y una política más conservadora se aleja más de los docentes, con cierta desconfianza hacia ellos, y confía más en un saber más académico. Y eso ha ido pasando en las diversas autonomías españolas y en otros contextos de diversos países. Y trajo un cierto desánimo de lo que apareció en la década de los años 80 y se había ido construyendo. Y todo ello se agravó con los recortes de la década del 2010, cerrando centros de profesores, recortando la formación de asesores y profesores, aumentando las exigencias hacia el profesorado, aumentando ratios y horarios y asumiendo un mayor papel técnico y burocrático. Desánimo, consternación y gran desconcierto que llevó a una vuelta hacia atrás aplicando el modelo técnico aplicacionista-transmisivo (de lecciones modelo de expertos, de manuales, de formación on-line, de nociones, ortodoxia, de competencias...); es decir, que se volvía a la orientación del formador/a hacia la solución de los problemas del profesorado, en lugar de la lucha que se iba llevando por un modelo de formación más regulativo, indagativo y reflexivo (investigación-acción, heterodoxia, modelos variados, inventiva didáctica...), donde es importante la persona, la identidad docente, el contexto, el profesional y la institución y no tanto la normativa, la zona de confort, la rutina y el adocenamiento. Aparece y revive la desprofesionalización y eso empeora la situación de la formación ya que aparece una gran desconfianza hacia ella.

Y la formación permanente ha ido recibiendo nuevos factores que la condicionan: nuevas tecnologías, cambios sociales y del alumnado, la caducidad del conocimiento y, por tanto, también a las instituciones que se dedican a ello, la creciente diversidad, multiculturalidad, conocimiento en otras instancias socializadoras, informes internacionales, leyes y normativas, etc., y también el avance de ciertas ideologías neoconservadoras con un determinado concepto de la formación y del papel del profesorado, con una propaganda mediática de volver a los enfoques tecnológi- 
cos, funcionalistas y burocratizantes. Pero todo ello no ha impedido que se hayan ido generando propuestas y alternativas para mejorar el papel del profesorado y su formación a lo largo de la vida personal, profesional e institucional.

\section{5. ¿QUÉ ALTERNATIVAS APARECEN DE MEJORA EN DESARROLLO A LO LARGO DE LA VIDA DOCENTE?}

Analizaremos algunas de las alternativas o proposiciones que la experiencia, la evidencia y la investigación nos muestran y nos ayudan a mejorar el desarrollo del profesorado. Algunas ya hace tiempo que están en la palestra de la formación tanto práctica como teóricamente y otras han ido apareciendo en los últimos decenios.

\section{A) El triángulo del desarrollo docente}

Una de las nuevas miradas es ver que el desarrollo docente no es únicamente desde la perspectiva profesional, sino también como desarrollo personal e institucional. En el profesorado será tan importante el desarrollo como persona (el bienestar docente provoca mejores resultados de aprendizaje), que como el desarrollo profesional (en cuanto que educador o educadora), y el institucional (las relaciones con los compañeros y el desarrollo conjunto en la institución educativa).

Un desarrollo personal, ya que el reconocerse de forma identitaria como docente permite interpretar mejor el trabajo docente, interactuar mejor con los otros, con el contexto que se vive día a día en los centros educativos, ya que las experiencias de vida del profesorado se relacionan con las tareas profesionales ya que la enseñanza requiere una implicación personal. Y una formación basada en la reflexividad será un elemento importante para analizar lo que son o creen ser y lo que se hace y cómo se hace. Ello nos lleva a concienciarse del papel personal del profesorado en los procesos de cambio y en la capacidad de generar cambios en sus contextos educativos y ello lo ha de fomentar la formación. Un profesor o profesora con malestar docente repercute en los procesos educativos. El desarrollo personal basado en un bienestar docente ayuda al docente de forma individual y colectiva a desarrollar un proceso de enseñanza-aprendizaje mucho más efectivo.

Por desgracia, la historia del profesorado es una historia de considerarle una persona dependiente y subsidiaria tratado hace tiempo por la literatura pedagógica (Popkewitz, 1996). En formación permanente, la consecuencia de este tipo de actuación ha ocasionado que el profesorado sea condenado a ser objeto de formación. Así, una persona, a quien se le suponía más conocimiento y saber (a veces mayor experiencia o jerarquía), adoctrinaba a un profesorado sobre la concepción implícita de su ignorancia y acatamiento. El cambio, en el futuro de la formación permanente, pasa por que el profesorado asuma ser sujeto de la formación y no un mero instrumento «objeto» en manos de otros, sea persona o administración.

$Y$ respecto al desarrollo institucional, o sea, en el centro educativo, es, hoy día, una herramienta fundamental en el desarrollo ya que el centro es la unidad 
del cambio. Históricamente, los procesos formativos se han tratado como un problema genérico y común que todos y todas podían resolver en las aulas, uniforme, estándar y que había que resolver mediante la solución genérica que los expertos o manuales aportaban. Ello comporta una gran descontextualización de la enseñanza y una gran desconfianza de la formación ya que para diferentes problemas educativos se sugería la misma solución, al margen de la personalidad, contexto geográfico, educativo y social concreto del profesorado y sin tener en cuenta cuáles eran las circunstancias que rodeaban la situación problemática específica. Ello ocasiona planes de formación con un sistema de formación estándar basado en cursos, seminarios o como se les quiera denominar, donde la racionalidad predominante era que los significados y las relaciones de las prácticas educativas eran transmitidas verticalmente. Se pretendía que cambiando al profesorado se cambiaría la educación y sus prácticas. Sin embargo, en la actualidad entrevemos que para cambiar la educación hemos de cambiar al profesorado y también los contextos experienciales donde este interactúa, donde se trabaja y se vive. El contexto en el que se dan las prácticas educativas, o sea, la propia institución educativa, que asume así una importancia decisiva. El desarrollo del profesorado tiene mucho que ver con el desarrollo colectivo y el contexto de la institución educativa.

\section{B) Formación UnidA A UN PROYeCto DE CAMbio y No AL REVÉS}

Otra alternativa un poco más antigua pero muy olvidada es unir la formación a un proyecto de trabajo en la institución educativa (OCDE-CER, 1985). Durante muchas décadas la estructura de racionalidad técnica de la formación permanente era realizar procesos de formación normalmente mediante cursos y después imaginar que el conocimiento adquirido serviría para realizar proyectos de cambio en la institución educativa. Y no es así, Es al revés. Se tienen que analizar las situaciones problemáticas de los centros, partiendo de las necesidades sentidas del profesorado, y recibir la formación necesaria para dar respuestas a esas necesidades mediante un proyecto de cambio. Es un cambio de binomio, primero proyecto de cambio y después formación para realizarlo adecuadamente y con rigor. No se puede ir de la teoría a la práctica sin conocer el contexto donde se realiza esa práctica. Lo que se lograba era que una minoría realizara experiencias de innovación en su aula, que desaparecía con ellos o ellas, y no una innovación institucional que quedara en el centro. El cambio de binomio proyecto-formación comporta una consolidación de innovaciones en los centros ya que parte de sus problemas y necesidades y se encuentran soluciones específicas que quedan de forma colectiva en los contextos educativos. Y la formación debería tener más esa orientación que la de formación-proyecto ya que el componente de la contextualización queda difuminado y la innovación se va perdiendo en el tránsito a la práctica educativa.

Es realizar una formación que partiendo de las complejas situaciones específicas educativas ayude a generar alternativas de cambio en el contexto donde se da la educación. Que ayude más que desmoralice o desmotive a quien no puede poner en práctica la solución del experto/a porque su contexto no le apoya o las diferen- 
cias son tantas que es imposible replicar la solución (a menos que esta sea rutinaria y mecánica). Por tanto, se reduce la formación estándar para acercar la formación al contexto del profesorado.

Cuando se introduce en la perspectiva formativa el componente de la diversidad y la contextualización como elemento imprescindible en la formación hay un cambio de mirada, ya que el desarrollo de cualquier profesional siempre tiene lugar en un contexto determinado. Todo ello comporta desencadenar una formación desde dentro, en la propia institución, donde se producen las situaciones problemáticas que afectan al profesorado. Y también introducir en el trabajo de proyectos de cambio e innovación la pausa formativa. Hacer pocas cosas y hacerlas bien. No por hacer muchas cosas se provoca mayor formación e innovación. Ello no implica realizar formación estándar para temas muy nuevos o que necesitan información para los docentes. Pero el salto a la implicación y la innovación se da en la formación en la institución educativa.

El profesorado crece y se desarrolla cuando adquiere un mayor conocimiento de la compleja situación en la cual su enseñanza se produce y lo puede compartir con los iguales. Por eso, se ha de unir en una amalgama teoría y práctica, experiencia y reflexión, acción y pensamiento, proyecto y formación, tanto para su desarrollo personal como profesional e institucional.

\section{C) LA HUIDA O CUESTIONAMIENTO DE PRÁCTICAS FORMATIVAS NO RIGUROSAS}

Es importante no olvidar la investigación y el rigor en las prácticas formativas. Si el modelo de formación ha de estar basado en la profesionalización y no en el espontaneísmo, la adquisición de conocimientos en los procesos formativos debe estar más basada en la investigación, en las evidencias de las disciplinas pedagógicas y promoviendo una conciencia profesional de marcado acento ético donde lo que hacemos ha sido comprobado y evaluado y provoca una mejora. Tener rigor y evidencias de lo que se hace es muy importante para evitar caer en errores o influencias de personas ajenas que imbuyen sus ideas a veces con otras intenciones que la mejora de la educación.

La investigación sobre la formación nos lleva a tener en cuenta la práctica real mediante un proceso de adquisición de teoría y de reflexión sobre la práctica. La formación basada en la práctica del docente no consiste en la aplicación de ciertos principios o máximas apriorísticos y de intuiciones, sino que más bien debería ser una reflexión activa y crítica, efectuada con el fin de resolver una situación problemática. Y eso ha sido la reivindicación durante muchos años: un profesorado práctico y reflexivo e investigador de su propia práctica. No podemos realizar prácticas formativas sin preguntarnos qué es lo que ocasionan, para qué sirven y en qué teorías, experiencias y prácticas investigadoras se basan.

Y, sobre todo, cuestionarse colectivamente lo que se hace y por qué se hace como un potente procedimiento para la formación gracias a la acción colaborativa que implica y al trabajo en equipo, mediante el cual el profesorado orienta, corrige y evalúa las situaciones problemáticas reales y toma decisiones para mejo- 
rar, analizar o cuestionar la práctica social y educativa (Monereo et al., 2002). O sea, se convierte en un investigador de su práctica. La investigación, individual y colectiva, nos ayudará a huir de realizar prácticas formativas basadas en la intuición o en las modas.

\section{D) LA COLABORACIÓN DOCENTE COMO POLÍTICA}

La enseñanza es un trabajo colectivo. Necesariamente colectivo. Aun podríamos decir más: la enseñanza se ha convertido en un trabajo imprescindiblemente colectivo.

La colaboración docente es un diálogo profesional y de interacción educativa compartida. Una formación sin propósitos de desarrollo colaborativo es una formación llevada al fracaso ya que provocará un gran individualismo, en aulas de puerta cerrada con un celularismo docente y sin compartir lo que se hace. Es necesario desarrollar un clima y una metodología formativa que sitúe al profesorado en situaciones de participación, de aceptar críticas, de tolerancia, de discrepancia, de hablar de todo, suscitando la inventiva y la capacidad de regularlo. La formación debería tener en cuenta el desarrollo de la actitud colaborativa y la tolerancia profesional como proceso imprescindible en la formación del profesorado. Esa formación permitirá salir de los propios límites, ya sean nuestros o impuestos por otros. No tener miedo a lo inesperado, a los cambios, a las nuevas ideas.

La formación sirve al desarrollo cuando propicia un aprendizaje en y desde la colegialidad participativa y no una colegialidad artificial (la colegialidad artificial viene a menudo provocada por la obligación externa de realizar ciertos trabajos que demandan un proyecto colectivo, pero sin el necesario proceso real de colaboración ni basado en necesidades reales) (Hergreaves, 1996). En la colaboración se desarrolla una responsabilidad compartida, el compromiso, la disposición a compartir la tarea compleja de la institución educativa, involucrando siempre la capacidad crítica y de revisión constante.

Colaborar quiere decir compartir con otros, dudas, experiencias, procesos vitales, contradicciones, problemas, éxitos, fracasos, etc. Hablar de todo. Son elementos importantes en el desarrollo personal, institucional y profesional del profesorado. El individualismo provoca el silencio y la afonía.

Y observar a los compañeros de forma colaborativa. La observación de la práctica docente es una estrategia importante para la mejora de la docencia. Es una práctica colaborativa centrada en el diseño de un proceso de enseñanza.

Hay una serie de modalidades y estrategias formativas importantes y destacadas en las que basar la formación permanente en la colaboración: la potenciación de grupos y redes colaborativos, las comunidades de formación, los grupos de proyectos, los grupos de intercambio de ideas o experiencias, los equipos autónomos de investigación sobre la práctica educativa, etc. Para ello es necesario potenciar en la cultura del profesorado el desarrollo de procesos autónomos colectivos en el trabajo docente, pero entendidos como una autonomía compartida y no como una mera suma de individualidades. 
La colaboración desarrolla una actitud crítica y revulsiva de las clases ideadas como celdas, los agrupamientos homogéneos bajo criterios no coherentes, la jerarquización dentro de las instituciones, la creciente especialización y la parcelación de la enseñanza que constriñen e impiden una forma de trabajar conjunta. Por el contrario, el mantenimiento y el conformismo en esas situaciones y estructuras legitiman y facilitan el continuar trabajando de un modo aislado.

\section{E) El trabajo DOCENTE CON LA COMUNIDAD}

Otro de los elementos importantes que ha ido apareciendo en el campo educativo es la imposibilidad, en ciertos contextos sociales, de partir del principio de que la enseńanza puede extraer de la exclusión social a la totalidad o a la mayoría de la población. Los actuales contextos sociales, familiares y económicos nos muestran claramente que sin la ayuda de la comunidad que envuelve la institución educativa es difícil, en contextos singulares, luchar contra la exclusión social.

Pero del mismo modo que el profesorado ha de asumir su papel en la estructura organizativa educativa, la comunidad, y sus diversos componentes, también tendrán que asumir el suyo. Será necesario compartir procesos educativos y formación, realizar conjuntamente una reflexión sobre qué es necesario cambiar y cómo en las instituciones para disminuir y desterrar la exclusión social. La formación conjunta con la comunidad se perfila, en los diversos contextos educativos y sociales, como una de las alternativas a la difícil problemática de exclusión social de una parte de la humanidad. El profesorado es un agente activo social y comunitario y la institución educativa ha de ir más allá del proyecto educativo creando un proyecto educativo comunitario.

En cuanto al trabajo conjunto con la comunidad que involucra a la institución educativa como esfera pública, se amplía la noción de escuela y aula y las posibilidades y funciones educativas de estos espacios. Hay un cambio en la organización y gestión. La comunidad no es tanto una estructura como un conjunto de prácticas (Bourdieu, 1991). Ya no se trata de la institución educativa como lugares entre las paredes, sino de cualquier espacio donde se establezca una relación educativa y, por lo tanto, comunicativa entre el alumnado, la comunidad que envuelve el centro y el profesorado. La comunidad se nutre de la comunicación y el diálogo que se establece con el profesorado. Habermas (1998) hablará de una comunidad comunicativa organizada por comunicación y no por autoridad, estado o rito. Esta perspectiva aumenta el grado de responsabilidad y autonomía del profesorado en su gestión educativa y destaca su papel activo en el proceso de cambio educativo y social.

Ser docente es asumir un carácter más relacional, más cultural-contextual y comunitario, donde adquiere importancia la interacción entre los compañeros y todas las personas vinculadas a la comunidad. El siglo xxi configura una nueva forma de hacer de profesor o profesora puesto que tiene que participar activa y críticamente en su contexto y transmitir a los futuros ciudadanos y ciudadanas unos valores y unas formas de comportamiento democrático, igualitario, respetuoso de la diversidad cultural y social, del medio ambiente, etc. 
La práctica educativa cambia únicamente cuando el profesorado la quiere modificar, y no cuando el formador u otro medio lo dice o lo pregona. La crisis del solucionador educativo en la formación del profesorado está ya abierta, a pesar de la larga tradición de su práctica formativa.

Poco a poco ha ido surgiendo la conciencia de que el formador o formadora debe asumir más un papel de práctico reflexivo, en un modelo más regulativo en el que será fundamental ayudar a analizar los obstáculos que encuentra el profesorado para acceder a un proyecto formativo que le ayude a mejorar. El formador ha de ayudar a saltar esos obstáculos, personales e institucionales, para que el profesorado encuentre la solución a su situación problemática en el contexto donde trabaja. En el futuro será más necesario disponer de formadores diagnosticadores que de solucionadores de problemas educativos ajenos.

La profundidad de ese cambio, desde el punto de vista del formador o formadora, tiene lugar cuando la formación pasa de ser un proceso de actualización desde arriba a convertirse en un espacio de reflexión, intercambio, de aprendizaje, formación horizontal e innovación para que el profesorado aprenda. Se pone más énfasis en la formación el aprendizaje del profesorado que en la enseñanza del mismo. Ello implica, por parte de los formadores, una visión global de lo que es la formación y, por supuesto, una nueva metodología de trabajo con el profesorado. No es lo mismo transmitir que compartir, ni actualizar que analizar, ni aceptar que reflexionar... No es lo mismo explicar mi teoría y mi práctica como formador que ayudar a descubrir la teoría implícita de las prácticas docentes para ordenarla, fundamentarla y revisarla; o para destruirla si fuera preciso. La formación tiene un papel crucial en ayudar a remover el sentido común pedagógico, las ideas preconcebidas que no funcionan, a recomponer el equilibrio entre los esquemas prácticos del profesorado que aplica en el proceso de enseñanza-aprendizaje y los esquemas teóricos que los sustentan. De ahí que la práctica formativa siempre es compleja, como todo proceso de educación y vida (Morin, 2001).

El formador o formadora en la formación permanente debe asumir más un papel de práctico colaborador, en un modelo más reflexivo en el que será fundamental crear espacios de aprendizaje para ayudar a que el profesorado encuentre «su» solución para acceder a un proyecto que le ayude a mejorar. Solo cuando el profesorado encuentra solución a su situación, la interioriza y la experimenta se da un cambio en la práctica educativa.

También la estructura organizativa de la formación permanente debería cambiar. Si durante mucho tiempo la organización de referencia fueron los centros de profesores o las instituciones de apoyo a la formación, lo que necesitará el profesorado en el futuro serán estructuras más flexibles, redes de formación, intercambio e innovación, aún más cercanas a las instituciones educativas y, por supuesto, introducir la formación dentro de las escuelas y los contextos. El modelo de entrenamiento mediante planes institucionales debe entrar en crisis para dejar paso a un modelo más indagativo y de desarrollo y mejora de la enseńanza mediante proyec- 
tos de cambio y de innovación donde el profesorado de un contexto determinado asuma el protagonismo que deben tener y sean ellos quienes planifiquen, ejecuten y evalúen su propia formación, con ayuda o sin ella.

Ello supone una orientación de la formación hacia un proceso de provocar una reflexión basada en la participación y mediante metodología formativa basada en casos, intercambio, debates, lecturas, trabajo en grupo, incidentes críticos, situaciones específicas, elaboración de proyectos, indagación de problemas prácticos, etc., y de reflexión crítica y propuestas a nuevas políticas formativas. Exige un planteamiento diferente de la formación, donde predomina el trabajo conjunto, la profesionalización rigurosa y la denuncia de procesos ineficaces y nuevas propuestas colectivas.

\section{G) El PRofesorado como sujeto de Formación}

El profesorado debe ser sujeto de formación y no objeto de ella. Históricamente ha sufrido un proceso donde ha predominado ser objeto de formación y ser afónico en la intervención educativa. Pasar a ser sujeto de formación es dar la voz y el protagonismo a los docentes. Es tener en cuenta la capacidad del profesorado de generar conocimiento pedagógico mediante su trabajo práctico en las instituciones educativas. Y donde la escuela es la unidad del cambio y no el profesorado individual o asilado (Senge, 2000), como en perspectivas formativas técnicas y horizontales. Creerse en la capacidad de generar conocimiento pedagógico significa empoderar al profesorado como sujeto de formación y una formación horizontal. Una formación que acepte la reivindicación de la subjetividad del profesorado, de la identidad docente como un dinamismo de forma de ver y transformar la realidad social y educativa y de la capacidad de producción de conocimiento, que permita complementar la identidad del sujeto docente con la identidad grupal (Prieto-Parra, 2004). Lograr una visión crítica de la enseñanza para analizar la postura, los imaginarios de cada uno delante de la enseñanza y el aprendizaje, que aliente a la confrontación de preferencias, actitudes y valores, donde prevalezca el encuentro, la colaboración, la participación, la experiencia y la reflexión sobre lo que se hace entre colegas como elemento fundamental en la relación educativa (Day, 2006). Poner encima de la mesa lo que se piensa para confrontarlo con lo que piensan los demás y llegar a acuerdos conjuntamente.

Participar como sujeto en la formación significa participar de una manera consciente, implicando la ética, los valores, la ideología que permite comprender a los demás, analizar sus posicionamientos y sus visiones específicas. También será necesario que la formación suponga una mejora profesional posible y que esté suficientemente explicitada y resulte comprensible. Ello no evita que la formación, en cuanto que proceso de cambio, siempre generará resistencias, pero estas tendrán un carácter de rechazo si la formación se vive como una imposición arbitraria, de sumisión de ideas de otros, aleatoria, no verosímil y poco útil. 
No podemos olvidar que la formación permanente del profesorado se mueve en un mundo cada vez más complejo. La complejidad del entorno y del contexto educativo repercute en los procesos formativos. Esa creciente complejidad social y educativa origina que la profesión docente y su formación se hacen, en concordancia, también más complejas. No hay soluciones viejas para problemas nuevos. Reconocer la complejidad del pensamiento, de los valores y de la práctica docente significa reconocer que, la educación como fenómeno social, político e ideológica es una red abierta y que esa abertura hace que, a veces, se pueden tomar decisiones sin reflexionar (o intuitivas o desesperadas) y que promover una formación que facilite la reflexión sobre la complejidad puede hacer que el profesorado sean mejores gestores de la enseñanza-aprendizaje y de las emociones que comportan una determinada forma de ver los procesos educativos.

Introducirse en la complejidad desde la mirada de la formación significa intentar entender y comprender lo que pasa dentro y fuera de la institución, salir de los límites que nos imponemos inconscientemente (Morin, 2001) y tener la capacidad de autoorganizarse como colectivo en redes de intercambio y buenas prácticas. También quisiera hacer constar que dar respuesta a la complejidad educativa no significa el desorden, el escepticismo y el relativismo del todo vale o que la complejidad nos ha dejado desnudos de orden, valores o de normas como algunos apologistas de la postmodernidad insinúan. $\mathrm{Al}$ revés, es abrirnos a nuevas ideas y no tener miedo a los cambios.

\section{I) Formación PERMANENTE Del PROFesorado y la (NO) CARRERA DOCENTE}

La carrera profesional vista como una parte importante de la profesionalización de los enseñantes es necesaria y bastante importante. La carrera docente es un elemento fundamental del desarrollo docente (Redon, Serra y Angulo, 2015) y la actual carrera del docente es plana, o sea, con poca promoción. Un profesorado sin carrera es un profesorado que puede no tener estímulos ya que no mejora su situación personal, profesional e institucional, ni motiva a mejorar el conocimiento y los saberes educativos, ni las habilidades y las actitudes de los trabajadores de la enseñanza.

Desde hace tiempo existen alternativas (Redon, Serra, Angulo, 2015). Por un lado, la posibilidad de promocionar saliendo de la institución educativa, y, complementariamente, es aquella que permite que los enseñantes se desarrollen dentro del sistema educativo práctico, o sea, dentro de las instituciones educativas. Si hubiera un estímulo de quedarse dentro de las instituciones educativas se permitiría que se quedaran muchas personas valiosas en los centros y ganaría el sistema educativo.

Una carrera profesional potencia un desarrollo docente más grande en los enseñantes, sobre todo en aquellos que quieren quedarse en las escuelas y continuar trabajando por la innovación. No puede ser que la promoción del profesorado se realice tan solo verticalmente, saliendo de su hábitat de trabajo, puesto que esto convierte la carrera profesional en una aspiración individual y no en un desarrollo 
colectivo y de mejora social. Y esto desmotiva a muchos enseñantes, que ven que son castigados promocionalmente para quedarse. Si no hay carrera docente se cae en el peligro de beneficiar al que no hace nada o se acomoda en los aspectos normativos y administrativos.

Tenemos que avanzar en todos los componentes que ayuden a mejorar la profesión docente y establecer un nuevo concepto de carrera profesional docente basado en el estímulo y el reconocimiento de los méritos acreditados. Seguro que ayudará a mejorar la calidad de la enseñanza.

Se necesita una formación que desarrolle al profesorado como persona, como profesional y como miembro de una institución educativa. Y ello requiere una formación inicial, una inducción laboral y permanente a lo largo de la vida, muy diferente. La formación debe servir para la mejora, no para el hastío, la rutina y la desprofesionalización, y también como revulsivo crítico frente al propio sistema formativo y educativo. 


\section{REFERENCIAS BIBLIOGRÁFICAS}

Alen, B. (2009). «El acompañamiento a los maestros y profesores en su primer puesto de trabajo. Profesorado". Revista de Currículum y Formación Del Profesorado, 13(1), 79-87. Consultado en http://digibug.ugr.es/bitstream/10481/9225/1/rev131ART5.pdf.

Bourdieu, P. (1991). El sentido práctico. Madrid: Taurus.

Bravo Reinoso, P. (2007). «Presupuestos epistemológicos para un entendimiento del sujeto de la educación», en El sujeto en la educación. Quito: Editorial Abya Yala.

DAY, C. (2006). Pasión por enseñar. La identidad personal y profesional del docente y sus valores. Barcelona: Narcea.

Eurydice (2002). «Temas clave de la educación en Europa». Volumen 3. Informe 1: Formación inicial y transición a la vida laboral. Educación Secundaria Inferior. Madrid: Ministerio de Educación, Cultura y Deporte. Consultado en https://sede.educacion.gob.es/publiventa/descargas.action?f_codigo=11393\&codigo.

Eurydice (2006). La evaluación de la calidad de la formación del profesorado europeo. Madrid: Ministerio de Educación y Ciencia. Consultado en file:///C:/Users/Francesc/Downloads/evaluacion_calidad_formacion_profesorado_europeo_eurydice.pdf.

Eurydice (2012). Cifras clave de la educación en Europa. Madrid: Ministerio de Educación, Cultura y Deporte. Consultado en https://sede.educacion.gob.es/publiventa/cifras-clave-de-la-educacion-en-europa-2012/ensenanza-union-europea/15849.

Eurydice (2013). Key Data on Teachers and School Leaders in European. Luxembourg: Publications Office of the European Union.

Friedman, I.A. (2004). «Directions in teacher training for low-burnout teaching», en E. Frydenberg (ed.), Thriving, surviving, or going under: Coping with everyday lives (pp. 305-326). Greenwich, Connecticut: Information Age Publishing.

González Sanmamed, M. y Fuentes, E.J. (2011). «El Prácticum en el aprendizaje de la profesión docente». Revista de Educación, 354, 47-70.

Guerra, N. y Lobato, C. (2015). «¿Con qué motivaciones y expectativas se acercan los futuros maestros a la profesión educativa?». INFAD Revista de Psicología, 1(1), pp. 331-342.

Habermas, J. (1998). Facticidad y validez. Trotta, Madrid.

Hergreaves, A. (1996). Profesorado, cultura y postmodernidad. Cambian los tiempos, cambia el profesorado. Madrid: Morata.

Imbernón, F. (2001). «Claves para una nueva formación del profesorado». Investigación en la escuela, n. ${ }^{\circ} 43$, pp. $57-66$.

IMBERnón, F. (2010). «Reflexiones globales sobre la formación y el desarrollo profesional del profesorado en el estado espańol y Latinoamérica». Revista Educar 30, 15-25.

Darling-Hammond, L. (2001). El derecho de aprender. Barcelona: Ariel.

Marina, J. et al. (2015). Consultado en file://C:/Users/Francesc/Downloads/libroblancomarina.pdf.

MEC (2012). Panorama de la Educación 2012: Indicadores de la OCDE. Consultado en https://sede. educacion.gob.es/publiventa/panorama-de-la-educacion-2012-indicadores-de-la-ocde/educacion-estadisticas-espana-europa/15844.

Morin, E. (2001). Los 7 saberes necesarios para la educación del futuro. Barcelona: Paidós. 
OCDE-CERI (1985). Formación profesores en ejercicio. Condición de cambio en escuela. Madrid: Narcea.

OCDE (2005). Teachers matter: attracting, developing and retaining effective teachers. París: OCDE.

Popkewitz, T.S. (1996). Formación del profesorado. Tradición, teoría y práctica. Valencia: Universidad de Valencia.

Prieto-Parra, M. (2004). «La construcción de la identidad profesional del docente. Un desafío permanente». Revista Enfoques Educacionales, 6 (1), 29-49.

Redon, S., Serra, L. y Angulo, F. (2015). Ley de la carrera docente: un punto de quiebre. Consultado en https://www.academia.edu/13245173/Ley_de_Carrera_Docente._Un_punto_ de_quiebre.

Senge, P. et al. (2000). Schools that learn. New York: Doubleday.

TALIS (2014). Informe TALIS 2013. Estudio internacional de la enseñanza y el aprendizaje. Consultado en http://www.mecde.gob.es/inee.

Talis (2018). Informe Talis 2018. Recuperado de http://www.educacionyfp.gob.es/inee/evaluacionesinternacionales/talis/talis-2018.html.

TARDif, M. (2004). Los saberes del docente y su desarrollo profesional. Madrid: Narcea.

Vezub, L. (2005). «Tendencias internacionales de Desarrollo Profesional Docente. La experiencia de México, Colombia, Estados Unidos y España». Buenos Aires: Direcciones Nacionales de Gestión Curricular y Formación Docente y de Investigación y Evaluación de la Calidad Educativa del Ministerio de Educación, Ciencia y Tecnología de la Argentina. 
